\author{
Abstracta Iranica \\ Abstracta Iranica Revue bibliographique pour le domaine irano-aryen \\ Volume 42-43 | 2021 \\ Comptes rendus des publications de 2019-2020
}

\title{
Carina Jahani. A Grammar of Modern Standard Balochi
}

\section{Agnes Korn}

\section{OpenEdition}

\section{Journals}

Édition électronique

URL : https://journals.openedition.org/abstractairanica/53055

DOI : 10.4000/abstractairanica.53055

ISSN : 1961-960X

Éditeur :

CNRS (UMR 7528 Mondes iraniens et indiens), Éditions de l'IFRI

\section{Référence électronique}

Agnes Korn, «Carina Jahani. A Grammar of Modern Standard Balochi », Abstracta Iranica [En ligne], Volume 42-43 | 2021, document 3, mis en ligne le 30 décembre 2021, consulté le 16 décembre 2022 URL : http://journals.openedition.org/abstractairanica/53055 ; DOI : https://doi.org/10.4000/ abstractairanica.53055

Ce document a été généré automatiquement le 16 décembre 2022.

Tous droits réservés 


\title{
Carina Jahani. A Grammar of Modern Standard Balochi
}

\author{
Agnes Korn
}

\section{RÉFÉRENCE}

Carina Jahani. A Grammar of Modern Standard Balochi. Uppsala : Uppsala Universitet, 2019, 292p. (Studia Iranica Upsaliensia 36), Accès libre.

1 Le baloutchi est une langue composée de trois groupes de dialectes qui divergent de manière importante et à tous les niveaux; il n'y a ni une forme standard ni une orthographe généralement acceptée. Cette situation pose évidemment un problème dans la perspective de la survie de la langue, qui n'a par ailleurs aucun statut officiel dans les pays où elle est parlée.

2 Le présent ouvrage se veut un outil pour remédier à cette situation en proposant une langue standard pour le baloutchi; il s'agit donc d'une grammaire prescriptive ou normative. Celle-ci est le fruit du travail d'un groupe de chercheurs et locuteurs du baloutchi à l'Université d'Uppsala, qui a en outre aussi produit un dictionnaire en ligne.

3 La base de cette grammaire est le baloutchi méridional, qui jouit d'un grand prestige parmi les baloutches et est aussi employé comme langue littéraire, bien que d'autres dialectes aient eux aussi leur littérature (par ex. la tradition épique transmise oralement en baloutchi oriental).

4 Visant le grand public, notamment les locuteurs du baloutchi ainsi que les personnes d'origine baloutche souhaitant se former à leur langue, le volume limite l'emploi de la terminologie linguistique au minimum et exemplifie chaque point abordé avec des exemples bien choisis.

5 Tous les exemples sont donnés à la fois en écriture arabo-persane et en écriture latine. Pour cette dernière, on utilise une orthographe élaborée avec des locuteurs : en effet, ceux-ci n'acceptaient pas d'employer des lettres qui ne se trouvaient pas sur un clavier. La solution fut donc un recours à des digraphes pour des consonnes; « dh, th, rh » 
indiquent les consonnes rétroflexes (notées $d, t, t, r$ dans des travaux descriptifs), « ch, sh, zh » sont mis pour $\check{c}, \check{s}$, $\check{z}$, et " gh, kh» représentent $\gamma, x$. Quant aux voyelles, dans un souci de limiter les diacritiques le plus possible, «i, é, á, ó, $u$ » ont été choisis pour les voyelles longues $(\bar{i} . \bar{e}, \bar{a}, \bar{o}, \bar{u})$ et « e, a, o » pour les voyelles courtes (prononcées $e, a$, o en Iran, mais $i, a, u$ dans les autres pays, ce qui est aussi leur valeur historique). L'orthographe arabo-persane suit l'un des styles employés au Pakistan, par ex. dans le grand dictionnaire "Sayad Ganj ", sans pour autant se soumettre à la particularité de cette orthographe en ce qui concerne l'emploi du « hamza » avec diacritiques (jugé trop compliqué par les locuteurs) pour les désinences de l'oblique $(-\bar{a})$ et du génitif $(-a y /-\bar{e})$ ainsi que pour « et » $(u)$.

6 Tout en étant une grammaire concise, le présent ouvrage survole tous les niveaux de la grammaire du baloutchi, y compris des sujets qui n'ont jusqu'ici que rarement été traités (ou pas du tout), comme l'intonation de la phrase et les noms composés. Au-delà de son objectif de codifier une langue standard, le volume contribue ainsi aussi à la description du baloutchi en général.

\section{AUTEURS}

\section{AGNES KORN}

CNRS, CeRMI, Paris 